\title{
Symmetric Circular Model Induced by Inverse Stereographic Projection On Double Weibull Distribution with Application
}

\author{
Phani Yedlapalli ${ }^{1}$, S.V.S.Girija ${ }^{2}$, A.V. Dattatreya Rao ${ }^{3}$ and G. V. L. N. Srihari ${ }^{4}$ \\ 1. Associate Professor of Mathematics Swarnandhra College of Engineering \& \\ Technology, Narsapur, India. \\ 2. Associate Professor of Mathematics, Hindu College, Guntur, India. \\ 3. Professor of Statistics, Acharya Nargarjuna University, Guntur, India. \\ 4. Associate Professor of Mathematics, Aurora Engineering College, \\ Bhongir, India.
}

\begin{abstract}
In practical life usually we come across angular or periodic data which needs distributions that are invariant of zero direction and sense of rotation for analysis. Inverse stereographic projection/ Bilinear transformation defined by a one to one mapping generate a class of probability distributions on unit circle that are flexible to analyse circular data. The probability density $f$ unction of many life testing models ranges from 0 to $\infty$. Aiming to construct new circular model by applying Inverse Stereographic Projection, we consider Double Weibull distribution and derive density, distribution and characteristic functions of new circular model coined by us Stereographic Double Weibull distribution. Also an attempt is made to study the mathematical aspects in evaluating trigonometric moments of new circular model and present the population characteristics. Goodness of fit to a live data is verified.
\end{abstract}

\section{KEYWORDS}

Characteristic function, Circular models, Inverse Stereographic Projection, Trigonometric moments, Unimodal.

\section{INTRODUCTION}

Wrapped versions of some life testing models on a unit circle were derived by Dattatreya Rao et al (2007) and Girija (2010). It is proposed that one way of constructing circular models by inducing inverse stereographic projection on linear models. Minh Do Le et al. (2003) proposed a new method of generating probability distributions on real line by applying bilinear transformation which maps every point on unit circle onto the point on real line. Dattatreya Rao et al. (2011) applied bilinear transformation on Cardioid distribution to generate Cauchy-Type models and also presented a differential approach to circular models. Various types of methods for constructing circular models are discussed in S.Rao Jammalamadaka et al. (2001) and Girija (2010). The double Weibull distribution has received considerable attention as an appropriate model in various fields. In particular, the double Weibull distribution is used to study the statistical aspects of re-emission spectra in a reverberation chamber. Balakrishna and Kocherla Kota (1985) introduced double Weibull distribution by reflecting Weibull Symmetrically to $(-\infty, 0]$ and studied linear estimation of location and scale parameters. He gave coefficients to find linear estimates of location and scale parameters for specified values of the shape parameter

DOI : 10.14810/ijscmc.2015.4106 
and tabulated there coefficients for complete samples up to size 10. Dattatreya Rao and Narasimham (1981) have extended the above work to various censoring schemes (left, right, symmetric \& double Censoring) tabulated the coefficients for sample sizes up to 20. Based on the approach of Minh et al. (2003) and Toshihiro Abe et al. (2010) circular models are derived by inducing inverse stereographic projection on double Weibull model. On the lines of Mardia and Jupp (2000) the characteristics of new circular model are evaluated and the graphs of pdf and cdf of stereographic double Weibull model are plotted using MATLAB. First four trigonometric moments are obtained in closed forms. Data set of 13 homing pigeons is modelled through the circular models by applying goodness of fit tests.

\section{METHODOLOGY OF INVERSE STEREOGRAPHIC PROJECTION}

Inverse stereographic projection which maps real line to unit circle is given

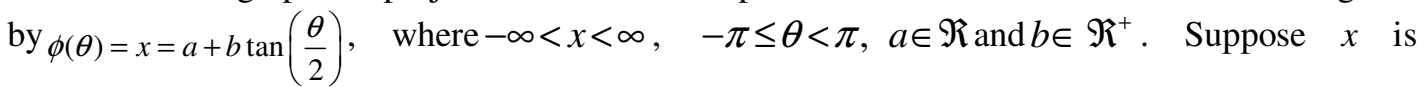
randomly chosen point on the interval $-\infty<x<\infty$. Let $F(x)$ and $f(x)$ denote cumulative distribution and probability density functions of the random variable $X$ respectively. Then $\phi^{-1}(x)=\theta=2 \arctan \left\{\frac{(x-a)}{b}\right\}$ by Minh et al. (2003) is a random point on the unit circle. Let $g(\theta)$ and $G(\theta)$ denote probability density and cumulative distribution functions of this random point $\theta$ respectively. Then $g(\theta)$ and $G(\theta)$ can be expressed in terms of $f(x)$ and $F(x)$ as following (Phani et al. (2012)).

Theorem 2.1: For $b>0$,

$$
\begin{aligned}
& g(\theta)=b\left(\frac{\sec ^{2}\left(\frac{\theta}{2}\right)}{2}\right) f\left(a+b \tan \left(\frac{\theta}{2}\right)\right) \\
& G(\theta)=F\left(a+b \tan \left(\frac{\theta}{2}\right)\right)
\end{aligned}
$$

\section{STEREOGRAPHIC DOUBLE WEIBULL DISTRIBUTION}

Here we recall the definition of Double Weibull Distribution (Dattatreya Rao et al. (1998)).

A random variable $X$ on the real line is said to have Double Weibull Distribution with scale parameter $\lambda>0$, location parameter $\alpha$ and shape parameter $c>0$, if its probability density and distribution functions of $X$ are respectively given by

$$
f(x)=\frac{c|(x-\alpha)|^{c-1}}{2 \lambda} \exp \left(-\frac{|(x-\alpha)|^{c}}{\lambda}\right)
$$

and 
International Journal of Soft Computing, Mathematics and Control (IJSCMC), Vol.4, No. 1,February 2015

$F(x)=(0.5)\left[1+\operatorname{sgn}(x)\left\{1-\exp \left(-\frac{|x-\alpha|^{c}}{\lambda}\right)\right\}\right.$,

where $c>0, \lambda>0$ and $-\infty<x, \alpha<\infty$.

By applying inverse stereographic projection defined by a one to one mapping $x=b \tan \left(\frac{\theta}{2}\right), b>0,-\pi \leq \theta<\pi$, which leads to a three parameter symmetric circular model on unit circle. We call this distribution as Stereographic Double Weibull distribution and it is denoted by $\operatorname{SDW}(\sigma, \mu, \mathrm{c})$.

\section{Stereographic Double Weibull Distribution}

A random variable $\theta_{S}$ on a unit circle is said to have Stereographic Double Weibull distribution with scale parameter $\sigma>0$, and location parameter $\mu$ and shape parameter $c>0$ denoted by $\operatorname{SDW}(\sigma, \mu, c)$, if its probability density and distribution functions are respectively given by

$$
g(\theta)=\frac{c}{4 \sigma} \sec ^{2}\left(\frac{\theta}{2}\right)\left|\left(\tan \left(\frac{\theta}{2}\right)-\mu\right)\right|^{c-1} \exp \left(-\frac{1}{\sigma}\left|\left(\tan \left(\frac{\theta}{2}\right)-\mu\right)\right|^{c}\right),
$$

and

$$
G(\theta)=(0.5)\left[1+\operatorname{sgn}\left(\left(\tan \left(\frac{\theta}{2}\right)-\mu\right)\right)\left\{1-\exp \left(-\left.\frac{1}{\sigma}\left(\tan \left(\frac{\theta}{2}\right)-\mu\right)\right|^{c}\right)\right\}\right],
$$

where $\sigma=\frac{\lambda}{b^{c}}>0, c>0$ and $-\pi \leq \theta, \mu=\frac{\alpha}{b}<\pi$.

Clearly

1. $g(\theta) \geq 0$ for every $-\pi \leq \theta<\pi$

2. $g(\theta+2 \pi p)=g(\theta), p \in \mathrm{Z}$ and

$3 \cdot \int_{-\pi}^{\pi} g(\theta) d \theta=1$

Plots of the probability density and distribution function of stereographic double Weibull distribution for various values of $\sigma, c$ and $\mu=0$ are presented here. 
International Journal of Soft Computing, Mathematics and Control (IJSCMC), Vol.4, No. 1,February 2015

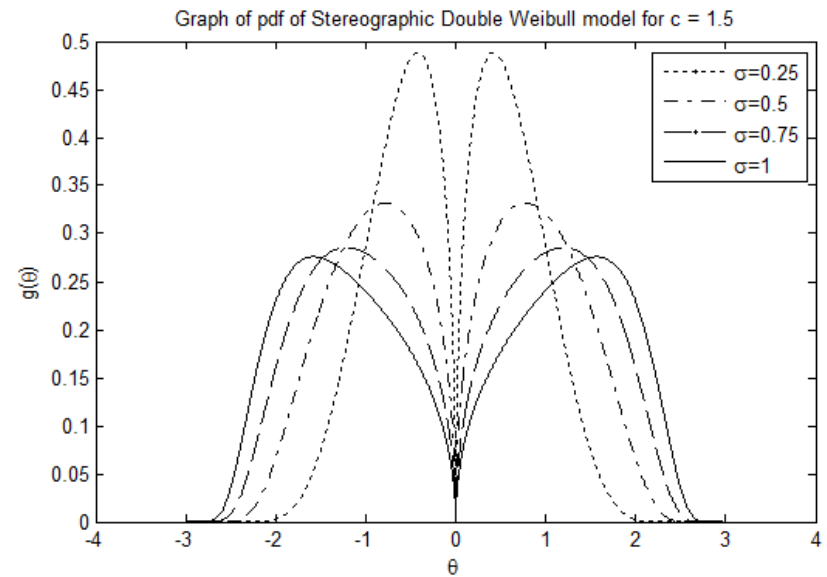

Figure-1

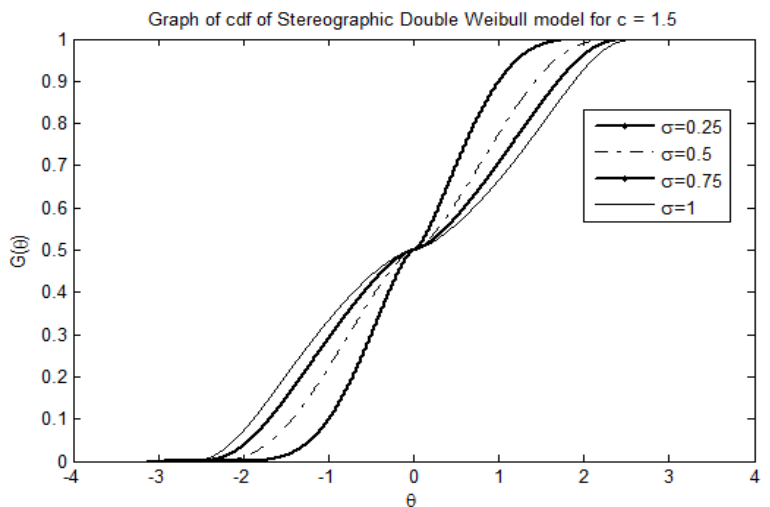

Figure-2

\section{THE CHARACTERISTIC FUNCTION OF STEREOGRAPHIC DOUBLE WEIBULL DISTRIBUTION}

The characteristic function of stereographic double Weibull model is given by

$$
\begin{aligned}
\Phi_{\theta_{s}}(p)= & \int_{-\pi}^{\pi} e^{i p \theta} g(\theta) d \theta \\
& =\int_{-\pi}^{\pi} e^{i p \theta} \frac{c}{4 \sigma} \sec ^{2}\left(\frac{\theta}{2}\right)\left|\tan \left(\frac{\theta}{2}\right)\right|^{c-1} \exp \left(-\frac{1}{\sigma}\left|\tan \left(\frac{\theta}{2}\right)\right|^{c}\right) d \theta \\
& =\frac{c}{2 \sigma} \int_{0}^{\pi} \operatorname{cosp} \theta \sec ^{2}\left(\frac{\theta}{2}\right)\left(\tan \left(\frac{\theta}{2}\right)\right)^{c-1} \exp \left(-\frac{1}{\sigma}\left(\tan \left(\frac{\theta}{2}\right)\right)^{c}\right) d \theta
\end{aligned}
$$

As this integral cannot be obtained analytically in closed form, we apply numerical integration of Gauss-Laguerre is used for the computation of the population characteristic for all integral values of $p$.

\section{Trigonometric Moments}

The moments of the distribution are given by $\varphi_{p}=\alpha_{p}+i \beta_{p}$, where $\alpha_{p}=E(\cos p \theta)$ and $\beta_{p}=E(\sin p \theta)$ being the $p^{\text {th }}$ order cosine and sine moments of the random angle $\theta$, respectively. Because the 
International Journal of Soft Computing, Mathematics and Control (IJSCMC), Vol.4, No. 1,February 2015

stereographic double Weibull distribution is symmetric about $\mu=0$, it follows that the sine moments are zero.

Theorem 4.1 Under the pdf of Stereographic Double Weibull distribution with $\mu=0$, the first four $\alpha_{p}=E(\cos p \theta), p=1,2,3,4$, re given as follows:

$$
\begin{aligned}
& \alpha_{1}=1-\frac{2}{\sigma} \sum_{n=0}^{\infty}(-1)^{n}(\sigma)^{\left(\frac{2 n+c+2}{c}\right)} \Gamma\left(\frac{2 n+c+2}{c}\right) \\
& \alpha_{2}=1+\frac{4}{\sigma} \sum_{n=0}^{\infty}(-1)^{n}(n+1)(\sigma)^{\left(\frac{2 n+c+4}{c}\right)} \Gamma\left(\frac{2 n+c+4}{c}\right)-\frac{4}{\sigma} \sum_{n=0}^{\infty}(-1)^{n}(\sigma)^{\left(\frac{2 n+c+2}{c}\right)} \Gamma\left(\frac{2 n+c+2}{c}\right) \\
& \alpha_{3}=1-\frac{16}{\sigma} \sum_{n=0}^{\infty}(-1)^{n}(n+1)(n+2)(\sigma)^{\left(\frac{2 n+c+6}{c}\right)} \Gamma\left(\frac{2 n+c+6}{c}\right)+\frac{48}{\sigma} \sum_{n=0}^{\infty}(-1)^{n}(\sigma)^{\left(\frac{2 n+c+4}{c}\right)} \Gamma\left(\frac{2 n+c+4}{c}\right) \\
& -\frac{18}{\sigma} \sum_{n=0}^{\infty}(-1)^{n}(\sigma)^{\left(\frac{2 n+c+2}{c}\right)} \Gamma\left(\frac{2 n+c+2}{c}\right) \\
& \alpha_{4}=1+\frac{64}{3 \sigma} \sum_{n=0}^{\infty}(-1)^{n}(n+1)(n+2)(n+3)(\sigma)^{\left(\frac{2 n+c+8}{c}\right)} \Gamma\left(\frac{2 n+c+8}{c}\right) \\
& +\frac{160}{\sigma} \sum_{n=0}^{\infty}(-1)^{n}(n+1)(\sigma)^{\left(\frac{2 n+c+1}{c}\right)} \Gamma\left(\frac{2 n+c+1}{c}\right) \\
& -\frac{128}{\sigma} \sum_{n=0}^{\infty}(-1)^{n}(n+1)(n+2)(\sigma)^{\left(\frac{2 n+c+1}{c}\right)} \Gamma\left(\frac{2 n+c+1}{c}\right) \\
& -\frac{32}{\sigma} \sum_{n=0}^{\infty}(-1)^{n}(\sigma)^{\left(\frac{2 n+c+2}{c}\right)} \Gamma\left(\frac{2 n+c+2}{c}\right)
\end{aligned}
$$

Proof: The proof is the process of using some transformations. For the first cosine moment, use the transformation $x=\tan \left(\frac{\theta}{2}\right), \cos \theta=1-\frac{2 x^{2}}{\left(1+x^{2}\right)}$ and then use the property of an even function. So we have the following integral $\alpha_{1}=1-\frac{2 c}{\sigma}\left\{\sum_{n=0}^{\infty}(-1)^{n} \int_{0}^{\infty} x^{(2 n+c+2)-1} e^{-\frac{x^{c}}{\sigma}} d x\right\}$.

The result $\alpha_{1}$ follows by the integral formula 3.478.1 (Gradshteyn and Ryzhik, 2007).

To obtain second cosine moment $\alpha_{2}$, we use the $\operatorname{transformation}_{x=\tan \left(\frac{\theta}{2}\right)}$, $\cos 2 \theta=1+\left(\frac{8 x^{4}}{\left(1+x^{2}\right)^{2}}\right)-\left(\frac{8 x^{2}}{\left(1+x^{2}\right)}\right)$ and the use the property of an even function. So we have the following integral

$$
\alpha_{2}=1+\frac{4 c}{\sigma} \sum_{n=0}^{\infty}(-1)^{n}(n+1) \int_{0}^{\infty} x^{(2 n+c+4)-1} e^{-\frac{x^{c}}{\sigma}} d x-\frac{4 c}{\sigma} \sum_{n=0}^{\infty}(-1)^{n} \int_{0}^{\infty} x^{(2 n+c+2)-1} e^{-\frac{x^{c}}{\sigma}} d x .
$$

The result follows by the same integral formula of $\alpha_{1}$. To obtain the third cosine moment $\alpha_{3}$, we use the transformation $x=\tan \left(\frac{\theta}{2}\right), \cos 3 \theta=1-\frac{32 x^{6}}{\left(1+x^{2}\right)^{3}}+\frac{48 x^{4}}{\left(1+x^{2}\right)^{2}}-\frac{18 x^{2}}{\left(1+x^{2}\right)}$ and the use the property of even function. We has the following integral 
International Journal of Soft Computing, Mathematics and Control (IJSCMC), Vol.4, No. 1,February 2015

$\alpha_{3}=1-\frac{2}{\sigma} \sum_{n=1}^{\infty}(-1)^{n-1} n\left\{-32 \int_{0}^{\infty} x^{6}\left(1+x^{2}\right)^{-3} e^{-\frac{n}{\sigma} x} d x+48 \int_{0}^{\infty} x^{4}\left(1+x^{2}\right)^{-2} e^{-\frac{n}{\sigma} x} d x-18 \int_{0}^{\infty} x^{2}\left(1+x^{2}\right)^{-1} e^{-\frac{n}{\sigma} x} d x\right\}$

The result follows by the same integral formula of $\alpha_{1}$.

To obtain the fourth cosine moment $\alpha_{4}$, we use the transformation ${ }_{x=\tan }\left(\frac{\theta}{2}\right)$, $\cos 4 \theta=1+\frac{128 x^{8}}{\left(1+x^{2}\right)^{4}}+\frac{160 x^{4}}{\left(1+x^{2}\right)^{2}}-\frac{256 x^{6}}{\left(1+x^{2}\right)^{3}}-\frac{32 x^{2}}{\left(1+x^{2}\right)}$ and the use the property of even function .we has the following integral

$$
\begin{aligned}
\alpha_{4}=1+\frac{32}{\sigma} \sum_{n=1}^{\infty}(-1)^{n-1} n\left\{8 \int_{0}^{\infty} x^{8}\left(1+x^{2}\right)^{-4} e^{-\frac{n}{\sigma} x} d x+10 \int_{0}^{\infty} x^{4}\left(1+x^{2}\right)^{-2} e^{-\frac{n}{\sigma} x} d x\right\} \\
-\frac{64}{\sigma} \sum_{n=1}^{\infty}(-1)^{n-1} n\left\{8 \int_{0}^{\infty} x^{6}\left(1+x^{2}\right)^{-3} e^{-\frac{n}{\sigma} x} d x-\int_{0}^{\infty} x^{2}\left(1+x^{2}\right)^{-1} e^{-\frac{n}{\sigma} x} d x\right\}
\end{aligned}
$$

The result follows by the same integral formula of $\alpha_{1}$.

Higher-order moments can be obtained similarly.

Using expressions in Mardia et al. (2000) and the first two trigonometric moments, the characteristics of stereographic double Weibull distribution are calculated numerically are presented in the table-1.

\begin{tabular}{|c|c|c|c|c|}
\hline & $\sigma=0.5$ & $\sigma=1.5$ & $\sigma=2$ & $\sigma=3.75$ \\
\hline Mean Direction $\mu_{0}$ & 0.0000 & 0.0000 & 0.0000 & 0.0000 \\
\hline Trigonometric $\quad \alpha_{1}$ & 0.9559 & 0.9327 & 0.8576 & 0.8077 \\
\hline Moments $\quad \alpha_{2}$ & 0.8556 & 0.7727 & 0.5365 & 0.4009 \\
\hline$\beta_{1}$ & 0.0000 & 0.0000 & 0.0000 & 0.0000 \\
\hline$\beta_{2}$ & 0.0000 & 0.0000 & 0.0000 & 0.0000 \\
\hline Resultant length $\rho$ & $\begin{array}{l}0.9559 \\
0.8556\end{array}$ & $\begin{array}{l}0.9327 \\
0.7727\end{array}$ & $\begin{array}{l}0.8576 \\
0.5365\end{array}$ & $\begin{array}{l}0.8077 \\
0.4009\end{array}$ \\
\hline Variance $\quad V_{o}$ & 0.0621 & 0.0673 & 0.1424 & 0.3283 \\
\hline Central trigonometric $\alpha_{1}^{*}$ & 0.9559 & 0.9327 & 0.8576 & 0.8077 \\
\hline Moments $\quad \alpha_{2}^{*}$ & 0.8556 & 0.7727 & 0.5365 & 0.4009 \\
\hline$\beta_{1}^{*}$ & 0.0000 & 0.0000 & 0.0000 & 0.0000 \\
\hline
\end{tabular}

Characteristics of Stereographic Double Weibull Distribution for $c=0.5$ 
International Journal of Soft Computing, Mathematics and Control (IJSCMC), Vol.4, No. 1,February 2015

\begin{tabular}{|c|c|c|c|c|}
\hline$\beta_{2}^{*}$ & 0.0000 & 0.0000 & 0.0000 & 0.0000 \\
\hline Skewness $\gamma_{1}^{o}$ & 0.0000 & 0.0000 & 0.0000 & 0.0000 \\
\hline Kurtosis & 10.6277 & 3.5252 & -0.2196 & -0.6656 \\
\hline $\begin{array}{l}\text { Circular } \\
\text { standard deviation } \sigma_{o}\end{array}$ & $\begin{array}{l}0.3004 \\
0.5585\end{array}$ & $\begin{array}{l}0.3733 \\
0.7181\end{array}$ & $\begin{array}{l}0.5543 \\
1.1159\end{array}$ & $\begin{array}{l}0.6536 \\
1.3521\end{array}$ \\
\hline
\end{tabular}

Table-1

Note: For $c=1$, Stereographic Double Weibull model reduces to Stereographic Double Exponential model SDEXP $(\sigma, \mu)$ (Dattatreya Rao et al. (2014)).

\section{Analysis of Homing Pigeons Data}

Goodness of fit tests is performed to verify whether the live data of 13 homing pigeons is fit to the Stereographic Double Weibull model. In homing pigeon data analysis, 13 birds were released singly in the Toggenburg Valley. Their vanishing angles are

$$
20^{\circ}, 135^{\circ}, 145^{\circ}, 165^{0}, 170^{\circ}, 200^{\circ}, 300^{\circ}, 325^{\circ}, 335^{\circ}, 350^{\circ}, 350^{\circ}, 350^{\circ}, 355^{0}
$$

Here goodness of fit tests are carried out. Using these $\theta_{i}{ }^{\prime} s$, the tests statistics of available tests are applied. The theoretical cut off points at $1 \%, 5 \%$ and $10 \%$ level of significance for size $n=13$ are taken form Devaraaj (2012), and the estimates of mean direction, scale parameter and shape parameter are $\mu=-0.2735, \sigma=0.2156$ and $c=1.456$ respectively.

The cut off points for a sample size $n=13$

\begin{tabular}{|l|l|l|l|}
\hline Level of Significance & $1 \%$ & $5 \%$ & $10 \%$ \\
\cline { 1 - 3 } Tests & $0.6950-2.1300$ & $0.7871-1.8691$ & $0.8268-1.7709$ \\
\hline $\begin{array}{l}\text { Kuiper's test } \\
\text { test }\end{array}$ & $0.0130-0.3334$ & $0.0187-0.2300$ & $0.0229-0.1932$ \\
\cline { 1 - 3 } $\begin{array}{l}\text { Rao's equal spacing } \\
\text { test }\end{array}$ & $1.1610-3.4940$ & $0.0396-0.7465$ & $1.0503-0.6263$ \\
\hline Rayleigh test & $0.0100-10.01$ & $0.0611-6.8871$ & $0.118-5.9786$ \\
\hline Hodges-Ajne test & $1.0000-6.0000$ & $1.0000-5.0000$ & $2.0000-5.0000$ \\
\hline Range test & $3.3630-5.4925$ & $3.7381-5.3957$ & $3.9930-5.3135$ \\
\hline Ajne test & $0.0290-1.0436$ & $0.0396-07465$ & $0.0503-0.6263$ \\
\hline
\end{tabular}

Table-2 
International Journal of Soft Computing, Mathematics and Control (IJSCMC), Vol.4, No. 1,February 2015

Statistic Value for seven tests to Stereographic Double Weibull Distribution for estimated values

\begin{tabular}{|l|l|l|}
\hline S.No & Test & Statistic \\
\hline 1 & Kuiper's test & 1.8439 \\
\hline 2 & Watson's $U^{2}$ test & 0.0079 \\
\hline 3 & Rao's equal spacing test & 2.5265 \\
\hline 4 & Rayleigh test & 1.4574 \\
\hline 5 & Hodge's Ajne test & 9 \\
\hline 6 & Range test & 4.4960 \\
\hline 7 & Ajne test & 0.2866 \\
\hline
\end{tabular}

Table-3

From these tests, it can be concluded that the homing pigeon data follows stereographic double Weibull distribution at $1 \%, 5 \%$ and $10 \%$ level of significance based on Rayleigh, Range, Rao's equal spacing and Ajne's tests and it also good fit at $1 \%$ and $5 \%$ with respect to Kuiper's test.

\section{ACKNOWLEDGEMENT}

The authors wish to thank the Editors and the anonymous referees for their comments and suggestions, which have greatly improved our manuscript.

\section{REFERENCES}

[1] Balakrishna, N and Kocherlakota, S. (1985), On the double Weibull distribution: order statistics and estimation, Sankhya, B-Series, 47, 2, pp.169-178.

[2] Dattatreya Rao, A.V., Ramabhadra Sarma, I and Girija, S.V.S.(2007), On Wrapped Versions of Some Life Testing Models, Comm Statist.,-Theor. Meth., 36, issue \# 11, pp.2027-2035.

[3] Dattatreya Rao, A.V., and Narasimham, V.L.(1989), Linear Estimation in Double Weibull distribution- Sankhya, B-Series, 51,1, pp.24-64.

[4] Dattatreya Rao, A.V., Girija, S.V.S., and Phani, Y.(2011), Differential Approach to Cardioid Distribution, Computer Engineering and Intelligent Systems, Vol. 2, No. 8, pp.1-6.

[5] Dattatreya Rao, A.V., Girija, S.V.S., and Phani, Y.(2014), New Circular Model Induced by Inverse Stereographic Projection on Double Exponential Model-Application to Birds Migration Data, Journal of Applied Mathematics, Statistics and Informatics(JAMSI), Vol.10, No.1, pp.5-17.

[6] Girija, S.V.S.(2010), Construction of New Circular Models, VDM-VERLAG, Germany.

[7] Gradshteyn, I.S., and Ryzhik, I.M.(2007), Table of Integrals, Series and Products, $7^{\text {th }}$ edition, Academic Press, San Diego.

[8] Jammalamadaka, S. Rao and Sen Gupta, A.(2001), Topics in Circular Statistics, World Scientific Press, Singapore.

[9] Mardia, K.V., and Jupp, P.E.(2000), Directional Statistics, John Wiley, Chichester.

[10] Minh, Do Le and Farnum, Nicholas R,(2003), Using Bilinear Transformations to Induce Probability Distributions, Comm Statist.,-Theor. Meth., 32, \# 1, pp.1-9.

[11] Phani, Y., S.V.S. Girija and A.V.Dattatreya Rao (2012), Circular Model Induced by Inverse Stereographic Projection on Extreme-Value Distribution, IRACST-Engineering Science and Technology: An International Journal (ESTIJ), Vol. 2, No.5, pp. 881-888.

[12] Toshihiro Abe, Kunio Shimizu and Arthur Pewsey,(2010), Symmetric Unimodel Models for Directional Data Motivated by Inverse Stereographic Projection, J. Japan Statist. Soc., Vol. 40, No.1, pp. 45-61. 